\title{
Les marqueurs de contraste au contraire, par contre et en revanche en français parlé et écrit, formel et informel
}

\author{
Jorina Brysbaert $^{1,2, *}$, et Karen Lahousse ${ }^{2}$ \\ ${ }^{1}$ Fonds de la Recherche Scientifique - Flandre (FWO), Egmontstraat 5, 1000 Bruxelles, Belgique \\ ${ }^{2}$ KU Leuven, Blijde-Inkomststraat 21, 3000 Leuven, Belgique
}

\begin{abstract}
Résumé. Dans cet article, nous présentons une analyse de la distribution des marqueurs de contraste au contraire, par contre et en revanche dans trois corpus, qui représentent trois registres différents : le français écrit formel (Le Monde), le français écrit informel (Yahoo Contrastive Corpus of Questions and Answers) et le français parlé (informel) (Discours sur la ville : Corpus de Français Parlé Parisien des années 2000). Nous constatons que les marqueurs contrastifs sont en général plus fréquents en français parlé qu'en français écrit formel, et que le français écrit informel semble former un registre "entre les deux». Notre analyse montre également que les trois marqueurs ont une préférence pour un registre particulier : en revanche est le marqueur le plus fréquent en français écrit formel, par contre s'utilise très souvent en français écrit informel et en français parlé informel, et au contraire est plus fréquent en français parlé qu'en français écrit. Nous observons aussi que la fréquence de par contre semble toujours être influencée par la critique normative à laquelle ce marqueur a longtemps été soumis. Le marqueur par contre n'est en effet presque jamais utilisé dans le corpus du français écrit formel.
\end{abstract}

\begin{abstract}
The contrastive markers au contraire, par contre and en revanche in spoken and written, formal and informal French. In this paper, we present an analysis of the distribution of the contrastive markers au contraire 'on the contrary', par contre 'on the other hand' and en revanche 'on the other hand' in three corpora, representing three different registers : formal written French (Le Monde), informal written French (Yahoo Contrastive Corpus of Questions and Answers) and (informal) spoken French (Discours sur la ville : Corpus de Français Parlé Parisien des années 2000). We observe that the contrastive markers are in general more frequent in spoken French than in formal written French, and that informal written French seems to form a register "in between". Our analysis also shows that the three markers have a preference for a certain register : en revanche is the most frequent marker in formal written French, par contre is very often used in informal written French and in informal spoken French, and au contraire is more frequent in spoken French than in written French. We also observe that the frequency of par contre still seems to be influenced by the normative criticism to which this marker has
\end{abstract}

\footnotetext{
* Corresponding author : jorina.brysbaert@kuleuven.be
} 
long been subjected. The marker par contre is indeed almost never used in the written formal French corpus.

\section{Introduction}

Dans cet article, nous étudions la distribution des marqueurs contrastifs au contraire (1), par contre (2) et en revanche (3) dans trois corpus, qui représentent trois registres différents : le français écrit formel (Le Monde) (3), le français écrit informel (YCCQA) (1) et le français parlé (CFPP2000) (2) :

(1) - Pour être " une vrai femme " faut il absolument devenir mère?

- Absolument pas au contraire je dirais même, car le fait d'être mère casse l'image même de la femme telle que l'homme la conçoit, c'est à dire une amante!!! (YCCQA) ${ }^{1}$

(2) spkl $^{2}:$ euh vous partez loin? + +

spk3 : alors en règle générale c'est + c'est toujours en France (mm) bon ça m'est arrivé d' voyager à l'étranger quelquefois + mais en règle générale c'est la France et euh j'pars beaucoup dans le dans l'Sud-est en fait + + dans l' Midi d'la France $q u o i+$ voilà

spk1 : d'accord et si vous pouviez déménager là?

spk3 : ah là par contre [rire] j'pense que + j'y réfléchirai quand même oui parce que je + c'est vrai qu' c'est c'est autre chose donc ça m' plaît aussi (mm) + donc euh j'me dis euh c'est + pourquoi pas on va dire pourquoi pas oui (CFPP2000)

(3) Au Maroc, si la radio et la télévision évoquent la violence qui secoue l'Algérie, c'est en termes neutres, sans prendre position. En Tunisie, en revanche, ils ne soufflent mot de la violence algérienne; ou lorsque l'ampleur d'une tuerie impose de lever le silence, ils relèguent l'information en fin de journal. (Le Monde)

Dans la littérature linguistique, beaucoup d'études ont été vouées à l'influence du registre sur la distribution de certains éléments linguistiques, en particulier des structures syntaxiques (e.g. Rowlett 2013), mais peu d'attention a été donnée à l'influence du registre sur la distribution des marqueurs contrastifs. Les marqueurs de contraste en français ont souvent été étudiés d'un point de vue plutôt sémantique et en outre, la plupart des analyses antérieures sont presque uniquement basées sur des données (limitées) du français (écrit) formel (e.g. Danjou-Flaux 1980; Lenepveu 2007; Masseron \& Wiederspiel 2003 ; Moeschler \& de Spengler 1981, etc.). La part du français parlé dans les corpus est généralement très limitée, et le registre du français écrit informel n'a même pas encore été pris en considération.

Pourtant, plusieurs linguistes ont déjà suggéré que les marqueurs contrastifs en français sont influencés par le registre. En particulier en ce qui concerne par contre et en revanche, il a souvent été mentionné que ces marqueurs se spécialisent pour un registre spécifique. La locution par contre a longtemps été critiqué par des puristes de la langue française et par conséquent, son emploi serait surtout fréquent en français parlé. Le marqueur en revanche constituerait sa "contrepartie soignée » et s'utiliserait surtout en français écrit formel. D'autres marqueurs, comme au contraire, semblent être moins contraints par le registre de langue. Toutefois, il manque encore une étude systématique de ces trois marqueurs, qui sont souvent considérés comme des (quasi-)synonymes (Danjou-Flaux 1980), dans plusieurs registres ${ }^{3}$.

L'article est structuré comme suit. Nous présentons d'abord les résultats des études antérieures sur les trois marqueurs contrastifs (section 2). Nous nous concentrons sur le marqueur par contre, dont l'emploi a longtemps été condamné (2.1), et sur le registre d'emploi des marqueurs au contraire, par contre et en revanche (2.2). Après une présentation des trois corpus (section 3), nous présentons les résultats de notre analyse 
(section 4). Nous donnons d'abord un aperçu général des données dans nos corpus (4.1), pour ensuite nous concentrer davantage sur le registre d'emploi de au contraire, par contre et en revanche (4.2) et sur l'effet de la critique normative sur la fréquence de par contre (4.3). Nous terminons avec quelques conclusions générales sur la distribution des trois marqueurs contrastifs (section 5).

\section{Analyses antérieures}

Dans cette section, nous donnons un aperçu des descriptions antérieures des marqueurs de contraste au contraire, par contre et en revanche. Une première sous-section (2.1) est consacrée au marqueur par contre, qui a longtemps été soumis à de la critique normative. Nous présentons d'abord les raisons pour lesquelles l'emploi de par contre a été condamné, pour ensuite expliquer comment la critique sur par contre s'est peu à peu affaiblie. Nous notons également que cette critique normative, bien qu'elle soit beaucoup moins prononcée de nos jours, semble toujours continuer à affecter la distribution de par contre. Dans une deuxième sous-section (2.2), nous nous concentrons sur la fréquence des marqueurs au contraire, par contre et en revanche dans différents registres. Nous présentons les résultats des analyses antérieures, qui suggèrent que par contre serait surtout fréquent en français parlé et que en revanche s'utiliserait le plus souvent en français écrit formel, tandis que au contraire aurait un emploi plus large. Nous remarquons toutefois que ces observations sont généralement basées sur des analyses d'un nombre de données trop limité.

\subsection{Par contre : un marqueur contrastif critiqué}

L'emploi de la locution par contre est fortement critiqué à partir du $18^{\text {ème }}$ siècle, notamment par Voltaire (Dictionnaire de l'Académie française 1992 (1) ; Grevisse 1964 ; Grevisse 1998 ; Grevisse \& Goosse 2008). Cette condamnation de par contre persiste jusqu'au $20^{\text {ième }}$ siècle, sous l'influence de quelques puristes et défenseurs de la langue française (p.ex. Hermant 1923 ; Joran 1928 ; Moufflet 1930 ; Vincent 1925) ${ }^{4}$. Le lexicographe Littré (1966) continue également à jeter le blâme sur le marqueur par contre dans son Dictionnaire de la langue française. Les raisons alléguées pour la condamnation de la locution par contre sont diverses. Certains linguistes, comme Capelovici (1990), Littré (1966) et Vincent (1925) la critiquent pour son «style commercial »". D'autres, comme Moufflet (1930) et Georgin (1966), s'indignent surtout du fait que par contre semble être une combinaison inappropriée de deux prépositions.

Si l'emploi de la locution par contre est longtemps désapprouvé, il importe aussi de souligner que la critique commence à s'affaiblir au cours du 20 ième siècle. Certains grammairiens et lexicographes attirent l'attention sur le fait que par contre est une locution assez courante, qui ne s'utilise pas seulement dans un langage quotidien mais qui figure également dans l'œuvre de différents auteurs renommés (Dictionnaire de l'Académie française 1992 (1) ; Doppagne 1966 ; Ducet 1967 ; Georgin 1966 ; Grand Larousse 1972 (2) ; Grevisse \& Goosse 2008 ; Hanse 1983) ${ }^{6}$. En outre, plusieurs linguistes mettent en question la préférence générale pour en revanche au détriment de par contre, car les deux marqueurs contrastifs ne semblent pas tout à fait avoir le même sens (Grand Robert 2001 (2) ; Grevisse 1964 ; Hanse 1983 ; Leeman-Bouix 1994). La locution en revanche s'utiliserait uniquement pour introduire un avantage (4), tandis que par contre pourrait introduire un avantage (4) ou un désavantage (5) :

(4) Jules a raté son examen d'anglais. \{Par contre / En revanche\}, il a réussi son examen de biologie. (notre exemple)

(5) Jules a réussi son examen de biologie. \{Par contre / En revanche\}, il a raté son examen d'anglais. (notre exemple) 
Par conséquent, il ne convient pas de complètement déconseiller l'emploi du marqueur par contre et de le remplacer par en revanche dans tous les contextes.

Malgré les efforts faits par différents auteurs pour démontrer que la critique sur par contre est injustifiée, la méfiance à l'égard de ce marqueur ne semble toujours pas entièrement avoir disparu. Ainsi, le Dictionnaire de l'Académie française (1992 (1) : 484) remarque au sujet de la locution par contre que «l'usage s'est établi de la déconseiller, chaque fois que l'emploi d'un autre adverbe est possible ». D'autres dictionnaires, comme le Dictionnaire du français contemporain (1966) et le Grand Larousse (1972 (2)) ne font pas vraiment la critique de par contre, mais mentionnent toutefois qu'il s'agit d'une locution déconseillée et condamnée. Le blâme qui a longtemps été jeté sur la locution par contre semble en outre avoir créé "une surnorme intériorisée " chez la plupart des francophones (Masseron \& Wiederspiel 2003 : 330). C'est ce que remarquent également Hamma \& Haillet (2002: 111-112) :

L'attitude des puristes à l'égard de par contre [...] continue de marquer des générations entières de locuteurs, notamment par le biais de la scolarisation ; les professeurs de français qui vont jusqu'à interdire à leurs élèves l'emploi de cette expression sont, aujourd'hui encore, sans doute plus nombreux qu'on ne pense - ce qui expliquerait la fréquence, chez beaucoup de sujets parlants, d'une sorte de réflexe d'hypercorrection les conduisant à considérer en revanche comme une variante "soignée " de par contre, qui relèverait, lui, d'un registre « familier » voir « relâché » et serait donc « à éviter ».

Dans notre analyse de corpus, nous étudierons si la critique qui a longtemps été lancée au sujet de par contre continue toujours à influencer la fréquence de ce marqueur dans différents registres. Nous nous concentrerons particulièrement sur le français écrit formel, et nous examinerons si l'affaiblissement de la critique se reflète par un emploi (de plus en plus) fréquent de par contre dans ce registre.

\subsection{Le registre d'emploi des marqueurs contrastifs au contraire, par contre et en revanche}

Selon la littérature linguistique, les marqueurs de contraste au contraire, par contre et en revanche ne s'utilisent pas avec la même fréquence dans tous les registres de la langue française. Cette section consiste en une présentation des analyses antérieures sur la fréquence de ces trois marqueurs. Il est toutefois important de souligner que les observations dans ces études sont souvent basées sur des données limitées, issues de corpus non comparables.

La locution en revanche semble s'employer le plus souvent en français écrit formel. Danjou-Flaux (1980 : 148) remarque en effet que le marqueur en revanche n'est presque jamais utilisé à l'oral, "du moins dans la langue courante », sans toutefois vérifier cette hypothèse sur la base d'un corpus. Ceci a été fait par Csüry (2001), qui analyse l'emploi de plusieurs marqueurs contrastifs dans un corpus qui contient cinq types de langage (environ 1.400 .000 mots au total) : (i) français écrit littéraire (romans écrits par Sartre, Maupassant et Flaubert), (ii) français écrit journalistique (Le Monde et Le Nouvel Observateur), (iii) français écrit politique/juridique (documents de l'Union européenne et de la Chambre des Communes du Canada), (iv) français parlé politique (Débats du Sénat du Canada) et (v) français parlé informel (Onze extraits de corpus de Blanche-Benveniste (1990)). Il est important de noter que les cinq sous-parties du corpus n'ont pas le même poids. Plus concrètement, les données du français écrit littéraire représentent $31 \%$ du corpus total, tandis que le français parlé informel ne constitue que $0,59 \% \mathrm{du}$ corpus total. $\mathrm{Ce}$ déséquilibre au niveau de la taille des différents sous-corpus a sans doute une influence sur les résultats de l'analyse de Csüry (2001) : certains sous-corpus, en particulier celui du français parlé informel, semblent être trop petits pour pouvoir donner une image 
représentative de la fréquence des marqueurs contrastifs dans un registre. En ce qui concerne en revanche, Csüry (2001) trouve 31 occurrences de ce marqueur dans son corpus. Il constate que en revanche est plus souvent utilisé en français écrit qu'en français parlé, ce qui confirme l'hypothèse de Danjou-Flaux (1980) (voir ci-dessus), et il précise que ce sont surtout les articles journalistiques et les documents politiques qui contiennent beaucoup d'occurrences du marqueur en revanche ${ }^{7}$. Ce résultat suggère donc que en revanche a une préférence pour le français écrit formel. Rappelons aussi que le marqueur en revanche est souvent considéré comme la contrepartie soignée de par contre (voir 2.1).

Tout comme en revanche, la locution par contre semble préférer un registre spécifique. Selon Danjou-Flaux (1980), par contre s'utilise presque uniquement en français parlé, mais elle ne vérifie pas cette hypothèse à l'aide d'une analyse de données authentiques. Dans leur analyse de corpus, Masseron \& Wiederspiel (2003) constatent que l'emploi de par contre est en effet assez rare à l'écrit. Cette observation n'est toutefois basée que sur « un corpus écrit d'une centaine d'emplois attestés » de par contre, contrairement à et au contraire, ce qui ne permet pas, selon nous, de tirer des conclusions sur la fréquence des marqueurs dans différents registres (Masseron \& Wiederspiel 2003 : 312). L'étude de Csüry (2001) montre également que par contre est employé plus fréquemment à l'oral qu'à l'écrit : le corpus de Csüry (2001) (voir ci-dessus) contient 44 occurrences de par contre, dont $90 \%$ figurent en français parlé. Finalement, la préférence de par contre pour le français parlé est corroborée par l'analyse de Bilger \& Cappeau (2003). Dans leur corpus de presse écrite (surtout Le Monde) (environ 1.000.000 mots), le marqueur par contre est quasi absent, tandis que dans leur corpus de français parlé en contexte politique (interviews, débats) (environ 300.000 mots), par contre est déjà plus souvent utilisé, et ce marqueur devient même très fréquent dans leur corpus de "français parlé divers " ${ }^{8}$ (interviews, explications, récits, etc.) (environ 1.000.000 mots) ${ }^{9}$. Les résultats de Bilger \& Cappeau (2003) suggèrent donc également que la fréquence de par contre serait beaucoup plus élevée en français informel (« français parlé divers ») qu'en français formel (français parlé en contexte politique). De façon assez surprenante, l'analyse de Csüry (2001) mène à une conclusion inverse. Dans le corpus de ce linguiste (voir ci-dessus), la locution par contre ne figure jamais en français parlé informel, alors qu'elle est très souvent utilisée en français parlé formel, représenté par des débats parlementaires canadiens ${ }^{10}$. Il importe toutefois de rappeler que le français parlé informel est sous-représenté dans le corpus de Csüry (2001) (seulement $0,59 \%$ du corpus total, voir ci-dessus), ce qui déforme sans doute les résultats de son analyse.

La distribution de la locution au contraire semble moins liée à un registre de langue particulier. Danjou-Flaux $(1980 ; 1983 ; 1984 ; 2003)$ ainsi que les auteurs du Grand Larousse (1972 (2)) et du Grand Robert (2001 (2)) ne mentionnent pas dans quels registres au contraire s'emploie le plus souvent, ce qui nous fait supposer que ce marqueur a un emploi assez large. Masseron \& Wiederspiel (2003) remarquent que, en comparaison avec par contre, le marqueur au contraire est souvent utilisé dans leur corpus écrit, sans toutefois mentionner le nombre d'occurrences exact. L'analyse de Csüry (2001) montre également que au contraire est surtout fréquent en français écrit formel : la plupart des 84 occurrences de au contraire dans son corpus figurent en français écrit journalistique et littéraire. Selon nous, il faut cependant prendre ce résultat avec précaution, étant donné que la part du français parlé informel dans le corpus de Csüry (2001) est très limitée et qu'il n'inclut pas le français écrit informel (voir ci-dessus).

Les différences de registre que nous avons présentées dans cette section sont résumées dans le Tableau 1. Deux aspects jouent un rôle important: le niveau de langue (formel versus informel) et le mode (écrit versus parlé). Le marqueur en revanche préfèrerait le français écrit formel, alors que par contre s'utiliserait surtout en français parlé. L'emploi de au contraire serait moins lié à un registre particulier, mais cette locution semble toutefois figurer le plus souvent en français écrit formel. Il importe aussi de souligner que la 
préférence des trois marqueurs pour un registre spécifique n'est pas vérifiée sur la base d'une analyse de corpus systématique. Les observations dans les analyses antérieures sont souvent basées sur un nombre de données limité, le registre du français écrit informel (p.ex. forum de discussion en ligne) n'est jamais pris en compte, et aucun linguiste ne compare la fréquence relative de chacun des trois marqueurs contrastifs au contraire, par contre et en revanche dans différents registres.

Tableau 1. Le registre d'emploi de au contraire, par contre et en revanche selon la littérature linguistique.

\begin{tabular}{|c|c|c|c|}
\hline Registre d'emploi & au contraire & par contre & en revanche \\
\hline $\begin{array}{c}\text { Niveau de langue } \\
\text { (formel/informel) }\end{array}$ & $\begin{array}{c}\text { formel }> \\
\text { informel (?) }\end{array}$ & $\begin{array}{c}\text { formel } / \\
\text { informel (?) }\end{array}$ & $\begin{array}{c}\text { formel }> \\
\text { informel }\end{array}$ \\
\hline $\begin{array}{c}\text { Mode } \\
\text { (écrit/parlé) }\end{array}$ & écrit > parlé (?) & parlé > écrit & écrit > parlé \\
\hline
\end{tabular}

Dans notre analyse de corpus, nous étudierons la fréquence de au contraire, par contre et en revanche dans trois corpus, qui représentent trois registres différents : le français écrit formel, le français écrit informel et le français parlé informel. La comparaison des fréquences dans ces trois corpus nous permettra de vérifier si les trois marqueurs ont une préférence marquée pour un registre particulier.

\section{Méthodologie}

Nous avons eu recours à trois corpus différents, qui ont été choisis en fonction du registre qu'ils représentent. Nous avons plus précisément inclus le français écrit formel (Le Monde), le français écrit informel (Yahoo Contrastive Corpus of Questions and Answers, désormais YCCQA) et le français parlé informel (Discours sur la ville : Corpus du Français Parlé Parisien des années 2000, désormais CFPP2000) ${ }^{11}$.

Le corpus journalistique Le Monde a été compilé par Piet Mertens (KU Leuven) et contient l'ensemble des articles qui sont parus dans le journal Le Monde en $1998 .^{12}$ Le corpus est consultable par le biais de CD-ROM d'archivage. L'extraction des occurrences de au contraire, par contre et en revanche a été effectuée par Piet Mertens.

Le YCCQA a été compilé par Hendrik De Smet (KU Leuven) et contient des productions langagières issues du site web https://answers.yahoo.com/, toutes datant de 2006 jusqu'à $2009^{13}$. Ce site web offre la possibilité de poster des questions et de répondre aux questions posées par d'autres utilisateurs. Les questions sont classées dans diverses catégories, selon leur sujet, par exemple « animaux », " grossesse et enfants », " politique et gouvernement», etc. Étant donné que les textes dans le YCCQA sont tous de type question-réponse, ils peuvent être considérés comme des "dialogues écrits ». Le YCCQA est en effet composé de productions langagières assez spontanées, qui ressemblent dans une certaine mesure à des conversations parlées. Il est toutefois important de souligner que les locuteurs qui participent à la conversation sont anonymes et ne se connaissent donc pas. Le recueil des occurrences des trois marqueurs dans le YCCQA, qui est disponible sous format TXT, a été effectué à l'aide du logiciel de concordance AntConc ${ }^{14}$.

Le CFPP2000 a été développé à partir de 2005 sous la direction de Sonia Branca-Rosoff (Paris 3-Sorbonne-Nouvelle). Il s'agit d'un corpus du français parlé informel, qui contient plus particulièrement des interviews faites à Paris et dans la banlieue de Paris. Les questions dans les entretiens concernent la vie des interviewés et «les rapports qu'ils entretiennent à leurs quartiers » (Branca-Rosoff et al. 2012 : 3). Dans la plupart des 
enregistrements, l'interviewer et le(s) interviewé(s) se connaissent, mais le degré de familiarité dépend d'une interview à l'autre. Le CFPP2000 est disponible sur le site web http://cfpp2000.univ-paris3.fr. Les transcriptions des interviews et les fichiers audios des enregistrements peuvent être consultées en ligne. Nous avons choisi de télécharger les transcriptions, c'est-à-dire nous les avons copiées sous format HTML pour ensuite les coller dans un éditeur de texte. Ainsi, nous avons obtenu une version TXT, que nous avons ouverte dans le logiciel AntConc, afin de pouvoir recueillir les occurrences de au contraire, par contre et en revanche.

Soulignons que dans le CFPP2000, il y a une " unité thématique », ce qui est moins le cas dans le YCCQA et Le Monde. Ceci implique que les contextes de conversation dans les trois corpus ne sont pas tout à fait identiques. Il importe toutefois de noter qu'il existe aussi une certaine variation à l'intérieur des différentes interviews dans le CFPP2000: les interviews durent généralement une à deux heures et portent sur de nombreux aspects de la vie des interviewé(e)s (à Paris), comme l'éducation, l'immigration, le transport, les loisirs, etc. Remarquons aussi que les données issues du corpus Le Monde (français journalistique) ont toutes plus ou moins le même degré de formalité, même si les textes ont été rédigés par plusieurs journalistes. Les deux autres corpus sont plus variables de ce point de vue : le degré de formalité des données dépend dans une certaine mesure du style personnel des différents locuteurs qui participent aux conversations. Selon nous, cela ne pose toutefois pas de problème en ce qui concerne la représentativité de nos corpus pour le registre dont ils relèvent, dans la mesure où le français informel, beaucoup plus que le français formel, se caractérise généralement par un degré de formalité plus variable. Il importe également de noter que nous considérons le YCCQA en quelque sorte comme le corpus « entre les deux ». Dans le modèle de Koch \& Oesterreicher $(1985,2007)$, le YCCQA pourrait être placé plus ou moins au milieu sur le continuum entre le langage parlé (i.e. language of immediacy) et le langage écrit (i.e. language of distance). ${ }^{15}$ En effet, pour plusieurs paramètres, le YCCQA se situe clairement vers le pôle language of immediacy: les textes dans ce corpus sont une sorte de « dialogues écrits », le choix des sujets de conversation est très libre et le langage est assez spontané. En ce qui concerne les autres paramètres de Koch \& Oesterreicher $(1985,2007)$, le YCCQA se rapproche plutôt du pôle language of distance: il s'agit de conversations entre des interlocuteurs anonymes, dans un cadre caractérisé par de la distance spatio-temporelle. Il s'ensuit également que, par rapport au YCCQA, le corpus Le Monde devrait être placé plus proche du pôle language of distance et le CFPP2000 plus proche du pôle language of immediacy.

\section{Résultats}

Dans cette section, nous présentons les résultats de notre analyse de corpus de au contraire, par contre et en revanche. Nous donnons d'abord un aperçu général de la fréquence des trois marqueurs dans les trois corpus (4.1). Nous constatons que les marqueurs contrastifs ne s'utilisent pas aussi fréquemment dans les différents corpus, ce qui suggère que la fréquence des marqueurs est influencée par le registre. Dans une deuxième sous-section, nous nous penchons davantage sur le registre d'emploi de au contraire, par contre et en revanche (4.2). Nous montrons que en revanche semble préférer le français écrit formel, que par contre figure presque uniquement en français informel, et que au contraire a un emploi plus large, mais s'utilise quand même le plus souvent en français parlé informel. Finalement, nous nous concentrons sur le marqueur par contre et l'effet de la critique normative sur sa fréquence (4.3). Nous observons que par contre est presque absent dans notre corpus de français écrit formel, mais cette quasi-absence ne semble toutefois pas être compensée par un emploi massif d'un des autres marqueurs contrastifs. 


\subsection{Aperçu général des données}

Le Tableau 2 offre un aperçu du nombre d'occurrences de au contraire, par contre et en revanche dans chacun des trois corpus. À titre de comparaison, nous avons également ajouté le nombre d'occurrences du marqueur contrastif de base mais. Les fréquences relatives dans le Tableau 2 ont été calculées par rapport au nombre de mots total dans le corpus en question ${ }^{16}$.

Tableau 2. Fréquences absolues et relatives de au contraire, par contre, en revanche et mais dans Le Monde, le YCCQA et le CFPP2000.

\begin{tabular}{|c|c|c|c|}
\hline Marqueur & $\begin{array}{c}\text { Le Monde } \\
25.700 .000 \text { mots } \\
\text { écrit (presse) }\end{array}$ & $\begin{array}{c}\text { YCCQA } \\
6.100 .000 \text { mots } \\
\text { écrit (forum de } \\
\text { discussion) }\end{array}$ & $\begin{array}{c}\text { CFPP2000 } \\
650.000 \text { mots } \\
\text { parlé (interviews) }\end{array}$ \\
\hline au contraire & $1481(0,058 \%)$ & $441(0,072 \%)$ & $68(0,105 \%)^{17}$ \\
\hline par contre & $46(0,002 \%)$ & $1520(0,249 \%)$ & $196(0,302 \%)$ \\
\hline en revanche & $1642(0,064 \%)$ & $292(0,048 \%)$ & $12(0,018 \%)$ \\
\hline mais & $40.628(1,581 \%)$ & $35.619(5,839 \%)$ & $6.640(10,215 \%)$ \\
\hline TOTAL & $43.797(1,704 \%)$ & $37.872(6,209 \%)$ & $6.916(10,640 \%)$ \\
\hline
\end{tabular}

Il s'ensuit du Tableau 2 que les marqueurs contrastifs s'utilisent en général le moins fréquemment en français écrit formel (Le Monde) (1,704\%o) et le plus fréquemment en français parlé (CFPP2000) (10,640\%). Le français écrit informel (YCCQA) (6,209\%o) se situe donc «au milieu »: les marqueurs contrastifs y sont plus fréquents qu'en français écrit formel (Le Monde), mais moins fréquents qu'en français parlé (CFPP2000).

En ce qui concerne au contraire, par contre et mais, la fréquence relative augmente en effet de gauche à droite dans le tableau : ils s'emploient moins souvent en français écrit formel (Le Monde) qu'en français écrit informel (YCCQA), et moins souvent en français écrit informel (YCCQA) qu'en français parlé (CFPP2000).

Pour en revanche, c'est tout à fait le contraire. Cette locution est plus fréquente en français écrit formel (Le Monde) qu'en français écrit informel (YCCQA), et plus fréquente en français écrit informel (YCCQA) qu'en français parlé (CFPP2000). Soulignons que, pour chaque marqueur, les fréquences les plus basses et les fréquences les plus élevées se trouvent dans les colonnes du corpus Le Monde et du CFPP2000. Ceci confirme donc que le YCCQA peut à juste titre être considéré comme le corpus « entre les deux » et représente aussi un registre « entre les deux ».

Il ressort également du Tableau 2 que, si on compare la fréquence des marqueurs à l'intérieur de chaque corpus, il existe une différence entre Le Monde d'un côté et le YCCQA et le CFPP2000 de l'autre. Dans Le Monde (français écrit formel), les marqueurs en revanche $(0,064 \%)$ et au contraire $(0,058 \%)$ sont clairement plus fréquents que par contre $(0,002 \%)$, tandis que dans le YCCQA (français écrit informel) et dans le CFPP2000 (français parlé), par contre $(0,249 \%$ - 0,302\%o) l'emporte nettement sur au contraire $(0,072 \%$ - $0,105 \%)$ et en revanche $(0,048 \%$ - $0,018 \%)$. Cependant, dans chacun des trois 
corpus, c'est le marqueur de base mais $(1,581 \%$ - 5,839\%o - 10,215\%o) qui s'emploie le plus souvent.

Cet aperçu général suggère que les différences de registre en ce qui concerne la fréquence des marqueurs contrastifs se révèlent importantes. Elles seront discutées de façon plus détaillée dans les sections suivantes (4.2 et 4.3).

\subsection{Le registre d'emploi des marqueurs contrastifs au contraire, par contre et en revanche}

Notre analyse de corpus montre que le marqueur en revanche s'utilise le plus souvent en français écrit formel (Le Monde) $(0,064 \%)$, et qu'il est plus fréquent que au contraire $(0,058 \%)$ et par contre $(0,002 \%)$ dans ce registre (voir Tableau 2). En français parlé, les locuteurs francophones n'optent que rarement pour en revanche $(0,018 \%)$ : les 12 occurrences de ce marqueur dans le CFPP2000 proviennent d'une seule interviewée et d'une seule enquêtrice. Nos résultats corroborent donc l'hypothèse de Danjou-Flaux (1980), selon laquelle en revanche ne s'utilise presque jamais à l'oral, ainsi que les observations de Csüry (2001), qui montre que en revanche est surtout fréquent dans des articles journalistiques et dans des documents politiques (voir 2.2).

En ce qui concerne par contre, il ressort du Tableau 2 que cette locution est très peu fréquente en français écrit formel $(0,002 \%)$ (voir 4.3 pour une discussion plus détaillée), mais s'emploie assez souvent en français écrit informel $(0,249 \%)$ et en français parlé $(0,302 \%)$. Dans ces deux derniers registres, par contre est nettement plus fréquent que au contraire $(0,072 \%$ - $0,105 \%)$ et en revanche $(0,048 \%$ - $0,018 \%)$. Ainsi, nos résultats confirment les études de Bilger \& Cappeau (2003), Csüry (2001), Danjou-Flaux (1980) et Masseron \& Wiederspiel (2003), dans lesquelles la locution par contre est généralement considérée comme une caractéristique du français parlé (voir 2.2). Notre analyse suggère aussi que le niveau de langue (formel/informel) pourrait avoir plus d'influence sur la distribution de par contre que le mode (écrit/parlé), dans le sens où le marqueur par contre s'utilise de préférence dans nos corpus de français plus informel, ce qui confirme également les résultats de Bilger \& Cappeau (2003). Remarquons toutefois qu'il importe de vérifier cette hypothèse sur un corpus de français parlé formel.

Comme il s'ensuit du Tableau 2, le marqueur au contraire s'utilise le plus fréquemment en français parlé (CFPP2000) $(0,105 \%)$, où il est deux fois plus fréquent qu'en français écrit formel (Le Monde) (0,058\%). Nos données ne confirment donc pas tout à fait l'hypothèse de Masseron \& Wiederspiel (2003), selon laquelle au contraire s'utilise souvent à l'écrit, et les résultats de Csüry (2001), qui observe que au contraire s'emploie surtout en français journalistique et littéraire. Remarquons aussi que, dans aucun des trois corpus, au contraire n'est le marqueur le plus fréquent, ce qui pourrait indiquer que sa préférence pour un registre particulier est peut-être moins prononcée que celle de en revanche et de par contre.

Le Tableau 3 offre un aperçu schématisé des résultats de notre analyse des différences de registre, et peut facilement être comparé avec le Tableau 1, qui représente les différences de registre telles qu'elles sont présentées dans la littérature linguistique. Les cas dans lesquels nos résultats se distinguent de ceux dans les études antérieures ont été soulignés.

Tableau 3. Le registre d'emploi de au contraire, par contre et en revanche dans nos corpus.

\begin{tabular}{|c|c|c|c|}
\hline Registre d'emploi & au contraire & par contre & en revanche \\
\hline $\begin{array}{l}\text { Niveau de langue } \\
\text { (formel/informel) }\end{array}$ & $\frac{\text { informel }>}{\underline{\text { formel }}}$ & $\frac{\text { informel ( }>}{\text { formel) }}$ & $\begin{array}{l}\text { formel }> \\
\text { informel }\end{array}$ \\
\hline
\end{tabular}




\begin{tabular}{|c|c|c|c|}
\hline $\begin{array}{c}\text { Mode } \\
(\text { écrit/parlé) }\end{array}$ & parlé $>$ écrit & parlé $>$ écrit & écrit $>$ parlé \\
\hline
\end{tabular}

\subsection{L'effet de la critique normative sur la fréquence du marqueur par contre}

Lors de la présentation des analyses antérieures (voir 2.1), nous avons signalé que beaucoup de francophones se méfient de par contre, à cause de la critique normative à laquelle cette locution a longtemps été soumise. Nous avons aussi remarqué qu'il semble donc logique que, selon la littérature linguistique, par contre ne s'utilise presque jamais en français écrit formel. Cette hypothèse est confirmée par la fréquence particulièrement basse du marqueur par contre dans Le Monde. En effet, comme le représente le Tableau 2, les journalistes de ce journal de qualité évitent clairement l'emploi de par contre (et témoignent donc d'une surnorme intériorisée) : il n'y a que 46 occurrences $(0,002 \%)$ de par contre dans le corpus Le Monde (voir ci-dessous pour une discussion de ces exemples), contre 1481 exemples $(0,058 \%)$ de au contraire et $1642(0,064 \%)$ de en revanche. La condamnation de par contre, quoique moins prononcée de nos jours, semble donc toujours avoir une influence considérable sur le registre d'emploi du marqueur.

Remarquons encore que, dans la littérature linguistique (voir 2.1), le marqueur en revanche est souvent traité comme le pendant soigné de par contre. Nos données ne peuvent toutefois pas vraiment confirmer cette hypothèse. Certes, en revanche s'utilise le plus souvent dans notre corpus de français écrit formel, mais la fréquence de en revanche dans Le Monde n'est pas aussi élevée $(0,064 \%)$ que celle de par contre dans le YCCQA $(0,249 \%$ ) et le CFPP2000 (0,302\%o) (voir Tableau 2). Le fait d'éviter l'emploi de par contre en français écrit formel ne semble donc pas provoquer un emploi «massif » du marqueur en revanche. Soulignons la ressemblance avec les résultats de l'analyse de Bilger \& Cappeau (2003). Ces auteurs constatent que par contre ne s'utilise presque pas dans leur corpus de français parlé en contexte politique et que «cette absence ne semble pas être compensée par un emploi "massif» de la tournure en revanche, généralement présentée comme un substitut acceptable » (Bilger \& Cappeau 2003 : 94-95). Il serait donc intéressant d'analyser quelles locutions ou quelles structures sont utilisées «à la place de » par contre en français écrit formel, pour marquer un même type de relation contrastive.

Pour terminer cette section sur par contre, nous nous concentrons encore sur les 46 occurrences de ce marqueur dans Le Monde (voir Tableau 2). Étant donné que les journalistes se gardent normalement d'utiliser la locution par contre, il nous semble intéressant d'examiner de plus près son contexte d'apparition dans ces 46 exemples. Nous avons d'abord vérifié si par contre s'y trouve surtout dans des phrases avec des " traces" du français parlé, c'est-à-dire dans le discours direct, i.e. des citations ou des interviews. Ceci pourrait indiquer que le journaliste n'a peut-être pas opté pour le marqueur par contre lui-même, mais qu'il a simplement repris les mots d'un certain locuteur, celui-ci ayant employé par contre dans un contexte moins formel. Notre recherche montre toutefois que ceci n'est pas vraiment le cas. Le marqueur par contre ne se trouve que sept fois dans le discours direct, dont quatre fois dans une citation (6) et trois fois dans un entretien (7), ce qui implique que la majorité des occurrences de par contre dans le corpus Le Monde n'apparaissent pas dans le discours direct :

(6) Il n'y a pas de malversation. Nous gardons toute notre confiance à Jean Blaise pour sentir et nous faire découvrir le monde. Il faudra, par contre, un contrôle de gestion plus rigoureux, explique l'adjoint à la culture, Yannick Guin. (Le Monde)

(7) Que vous inspire un nouveau projet de loi sur l'audiovisuel ? Je ne sais pas ce qu'il y aura dans ce projet de loi. [...] D'autant que l'un de nos credo est de n'être dominant dans aucun de nos secteurs. Aucune de nos activités ne dépasse 25\% à 
$30 \%$ de parts de marché. Ce qui est un problème, par contre, c'est qu'un journal télévisé fasse plus de 50\% d'audience. (Le Monde)

Nous avons ensuite examiné si par contre introduit plus souvent un "désavantage ", i.e. un élément négatif, qu'un "avantage », i.e. un élément positif, dans le corpus Le Monde. Selon certains linguistes, en effet (voir 2.1), la locution en revanche s'utiliserait uniquement pour introduire un avantage, alors que par contre pourrait introduire un avantage ou un désavantage. Il serait donc possible que les journalistes optent parfois pour par contre, au lieu de en revanche, afin de pouvoir introduire un élément négatif. Les résultats de notre analyse ne corroborent toutefois pas complètement cette hypothèse. Dans 18 des 46 cas, par contre introduit un élément négatif (8), mais le corpus contient également 15 exemples dans lesquels par contre est utilisé pour introduire un élément positif (9) et 13 exemples dans lesquels le marqueur exprime une relation plus ou moins neutre, i.e. l'élément introduit par par contre n'est ni nettement positif ni nettement négatif (10) :

(8) Cette position a été approuvée par les groupes politiques de droite, mais également par les élus socialistes qui participent, depuis 1995, à l'exécutif de la Courly. Elle a par contre été repoussée par les groupes FN, Gauche alternative et radical (Verts et ex-PC). (Le Monde)

(9) Gérald de Roquemaurel a indiqué qu'il n'était pas intéressé par la presse professionnelle ou économique d'Havas. Il serait prêt par contre à reprendre les journaux gratuits de la Comareg, filiale d'Havas, dans la région Provence-AlpesCôte d'Azur, si ceux-ci étaient à vendre. (Le Monde)

(10) Quel que soit le verdict des Lords britanniques, mercredi 25 novembre, chacun est convaincu qu'une page de l'histoire chilienne a été tournée. Personne, par contre, ne s'aventure à en prédire les conséquences. (Le Monde)

Ces résultats n'indiquent toutefois pas nécessairement que par contre commence à «s'intégrer » en français écrit formel. Afin de creuser l'emploi de par contre dans ce registre, il faudrait, par exemple, analyser ce marqueur dans différentes années du journal Le Monde ou dans différents types de journaux et de revues ${ }^{18}$. Ainsi, il serait possible de découvrir si la fréquence de par contre en français écrit formel augmente d'année en année ou si elle reste plutôt stable, et s'il y a des différences de fréquence entre différents types de français écrit formel.

\section{Discussion et conclusion}

Dans cet article, nous avons examiné la fréquence des marqueurs contrastifs au contraire, par contre et en revanche dans trois corpus, qui représentent trois registres différents : le corpus Le Monde (français écrit formel), le YCCQA (français écrit informel) et le CFPP2000 (français parlé (informel)). Notre analyse révèle que les marqueurs contrastifs s'utilisent en général plus souvent en français parlé qu'en français écrit informel, et plus souvent en français écrit informel qu'en français écrit formel. La locution en revanche présente toutefois une tendance inverse : elle est plus fréquente en français écrit formel qu'en français écrit informel, et plus fréquente en français écrit informel qu'en français parlé. Il est aussi important de souligner que le YCCQA (français écrit informel) est toujours le corpus «entre les deux» et semble donc représenter un registre «entre les deux » : aucun des trois marqueurs contrastifs ne s'emploie le plus souvent dans ce corpus.

Notre analyse montre également que les marqueurs au contraire, par contre et en revanche semblent se spécialiser pour un registre de langue spécifique. La locution par contre est très fréquente dans le corpus du français écrit informel et dans le corpus du français parlé informel. Ainsi, notre étude corrobore l'hypothèse dans la littérature linguistique que par contre est typique du français parlé, mais suggère en même temps que le niveau de langue (i.e. informel) a peut-être plus d'influence que le mode (i.e. parlé). La 
locution en revanche préfère s'utiliser en français écrit formel, où elle est plus fréquente que au contraire et par contre, ce qui confirme les études antérieures. En ce qui concerne au contraire, notre analyse montre que ce marqueur s'emploie deux fois plus souvent en français parlé qu'en français écrit formel, ce qui ne corrobore pas l'hypothèse des études antérieures selon laquelle ce marqueur serait surtout fréquent à l'écrit. Remarquons toutefois que au contraire semble avoir un emploi assez général, comme le suggère également la littérature linguistique : dans aucun de nos trois corpus, il n'est le marqueur le plus fréquent.

Dans cet article, nous nous sommes également concentrées sur par contre et l'effet de la critique normative sur la fréquence de cette locution. Nous avons constaté que le marqueur par contre est presque absent dans le corpus Le Monde, ce qui suggère que la critique qui a longtemps été lancée au sujet de par contre a toujours une grande influence sur le registre d'emploi de ce marqueur. Cette quasi-absence de par contre dans le registre du français écrit formel ne semble toutefois pas être compensée par un emploi plus fréquent d'un autre marqueur, comme en revanche, qui est souvent considéré comme l'alternative soignée. Remarquons aussi que, dans les quelques exemples où par contre figure dans le corpus Le Monde, il ne s'agit que rarement d'une représentation du discours direct, i.e. du français parlé, et le marqueur introduit presque assez fréquemment un élément positif que négatif. Il ne semble donc pas complètement exclu d'opter pour par contre dans un contexte formel à l'écrit, même dans des cas où l'emploi de en revanche serait tout à fait acceptable.

Selon nous, il serait également intéressant d'étudier la distribution de par contre dans d'autres corpus du français écrit formel (autres journaux/revues ou corpus nonjournalistiques), pour creuser l'effet de la critique normative sur l'emploi actuel de ce marqueur. En outre, il pourrait être utile de vérifier la fréquence des marqueurs au contraire, par contre et en revanche dans d'autres types de corpus, en particulier dans un corpus de français parlé formel, pour analyser davantage l'importance du niveau de langue (formel/informel) et du mode (écrit/mode) sur la distribution des trois marqueurs.

\section{Références bibliographiques}

Anthony, L. (2014). AntConc Version 3.4.4 [Computer Software]. Tokyo : Waseda University. http://www.laurenceanthony.net/.

Bilger, M. \& Cappeau, P. (2003). Les emplois de contre dans les corpus de français parlé et de presse écrite. In P. Péroz (ed.), Contre : Identité sémantique et variation catégorielle (Recherches Linguistiques 26), 91-111. Metz : Université de Metz.

Branca-Rosoff, S., Fleury, S., Lefeuvre, F. \& Pires, M. (2012). Discours sur la ville : Corpus de Français Parlé Parisien des années 2000 (CFPP2000). http://cfpp2000.univparis3.fr/CFPP2000.pdf.

Capelovici, J. (1990). Le français sans fautes : Répertoire des erreurs les plus fréquentes de la langue écrite et parlée. Paris : Acropole.

Csüry, I. (2001). Le champ lexical de mais : Étude lexico-grammaticale des termes d'opposition du français contemporain dans un cadre textologique (Studia Romanica de Debrecen, Series Linguistica 7). Debrecen : Kossuth Egyetemi Kiadó.

Danjou-Flaux, N. (1980). Au contraire, par contre, en revanche : Une évaluation de la synonymie. In Synonymies (Bulletin du Centre d'Analyse du Discours 4), 123-148. Lille : Presses universitaires de Lille.

Danjou-Flaux, N. (1983). Au contraire, connecteur adversatif. In Connecteurs pragmatiques et structure du discours (Cahiers de Linguistique Française 5), 275-303. Genève : Université de Genève. 
Danjou-Flaux, N. (1984). Au contraire comme opérateur d'antonymie dans les dialogues. In P. Attal \& C. Muller (eds.), De la syntaxe à la pragmatique (Lingvisticæ Investigationes Supplementa 8), 75-94. Amsterdam \& Philadelphia : John Benjamins Publishing Company.

De Smet, H. (2009). Yahoo Contrastive Corpus of Questions and Answers. Leuven : Department of Linguistics, University of Leuven.

Dictionnaire de l'Académie française. (1992). Paris : Imprimerie nationale.

Dictionnaire du français contemporain. (1966). Paris : Librairie Larousse.

Doppagne, A. (1966). Trois aspects du français contemporain. Paris : Librairie Larousse.

Ducet, M. (1967). Comment parler correctement et aisément : Du bon usage de la conversation et de la parole en public et en privé. Soissons : La diffusion nouvelle du livre.

Dürscheid, C. (2016). Nähe, Distanz und neue Medien. In H. Feilke \& M. Hennig (eds.), Zur Karriere von 'Nähe und Distanz'. Rezeption und Diskussion des Koch-Oesterreicher-Modells, 357-385. Berlin : De Gruyter.

Flaux, N. (2003). Au contraire (de) et le sens de contre. In P. Péroz (ed.), Contre : Identité sémantique et variation catégorielle (Recherches Linguistiques 26), 289-309. Metz : Université de Metz.

Georgin, R. (1966). Le code du bon langage. Paris : Les éditions sociales françaises.

Grand Larousse de la langue française. (1971-1978). Paris : Librairie Larousse.

Grevisse, M. (1964). Problèmes de langage. Gembloux : Duculot.

Grevisse, M. (1998). Le français correct : Guide pratique. Paris : Duculot.

Grevisse, M. \& Goosse, A. (2008). Le bon usage : grammaire française. Bruxelles : De Boeck.

Hamma, B. \& Haillet, P.P. (2002). Par contre : Un type particulier de dynamique discursive. Linx, 46, 103-113.

Hanse, J. (1983). Nouveau dictionnaire des difficultés du français moderne. Paris : Duculot.

Hermant, A. (1923). Xavier ou les entretiens sur la grammaire française. Paris : Le livre.

Joran, T. (1928). Les manquements à la langue française : Tournures et locutions vicieuses méthodiquement classées et redressées. Paris : Beauchesne.

Koch, P. \& Oesterreicher, W. (1985). Sprache der Nähe - Sprache der Distanz: Mündlichkeit und Schriftlichkeit im Spannungsfeld von Sprachtheorie und Sprachgeschichte. Romanistisches Jahrbuch, 36, 15-43.

Koch, P. \& Oesterreicher, W. (2007). Schriftlichkeit und kommunikative Distanz. Zeitschrift für Germanistische Linguistik, 35(3), 346-375.

Leeman-Bouix, D. (1994). Les fautes de français existent-elles? Paris : Éditions du Seuil.

Le Grand Robert de la langue française. (2001). Paris : Dictionnaires Le Robert - VUEF.

Lenepveu, V. (2007). Toutefois et néanmoins, une synonymie partielle. Syntaxe et Sémantique, 8, 91106.

Littré, É. (1966). Dictionnaire de la langue française. Paris : Gallimard - Hachette.

Masseron, C. \& Wiederspiel, B. (2003). Contrastivité adverbiale : Au contraire, contrairement à, par contre. In P. Péroz (ed.), Contre : Identité sémantique et variation catégorielle (Recherches Linguistiques 26), 311-341. Metz : Université de Metz.

Moeschler, J. \& de Spengler, N. (1981). Quand même : De la concession à la réfutation. Cahiers de Linguistique Française, 2, 93-112.

Moufflet, A. (1930). Contre le massacre de la langue française. Toulouse : Privat-Didier. 
Rowlett, P. (2013). Do French speakers really have two grammars? Journal of French Language Studies, 23(1), 37-57.

Vincent, C. (1925). Le péril de la langue française : dictionnaire raisonné des principales locutions et prononciations vicieuses et des principaux néologismes. Paris : De Gigord.

${ }^{1}$ Remarquons que les exemples issus des trois corpus ont été reproduits tels quels, ce qui implique que nous n'avons pas corrigé les erreurs de langue.

2 Dans cet exemple qui fait partie d'une interview, «spk1» réfère à l'interviewer et « spk3 » à un des interviewés.

3 Pour des raisons d'espace, nous ne pouvons pas prendre en considération plus de marqueurs contrastifs dans cet article (voir p.ex. Csüry (2001) pour une comparaison de plusieurs marqueurs de contraste).

${ }^{4}$ Ainsi, Joran (1928 : 108) qualifie la locution par contre d' " association de mots barbare, qui sera avantageusement remplacée par en revanche, en retour ». L'emploi de par contre est également désapprouvé par Vincent (1925: 42), qui estime que ce marqueur « offre [...] un sens louche », et par Moufflet (1930 : 174-175), qui conseille de substituer en revanche à par contre parce que «l'accumulation des mots invariables n'est pas esthétique ». Le puriste Hermant (1923 : 162) trouve que l'emploi de par contre donne l'impression d'être " né dans une arrière-boutique ».

${ }^{5}$ Selon Grevisse (1964: 108), le style commercial « prend assez communément une valeur péjorative et méprisante ».

${ }^{6}$ Le grammairien Grevisse $(1964 ; 1998$; Grevisse \& Goosse 2008) énumère à plusieurs occasions toute une série d'extraits dans lesquels des écrivains connus, comme Stendhal, France, Proust, Montherlant etc., emploient le marqueur par contre.

${ }^{7}$ Remarquons que Csüry (2001) ne mentionne pas le nombre d'occurrences exact par type de corpus.

${ }^{8}$ Nous reprenons ici le terme utilisé par Bilger \& Cappeau (2003).

${ }^{9}$ Soulignons que Bilger \& Cappeau (2003) ne calculent pas la fréquence relative de par contre sur la base du nombre de mots total dans chaque corpus.

${ }^{10}$ Csüry (2001 : 154) remarque que ce résultat ne suggère pas qu'il existe une différence régionale en ce qui concerne l'emploi de par contre, parce que son "expérience personnelle contredit cette hypothèse ».

${ }^{11}$ Remarquons que nous n'avons pas étudié la fréquence des trois marqueurs contrastifs dans le registre du français parlé formel, pour des raisons pratiques. Il s'est avéré impossible de facilement avoir accès à un corpus de français parlé formel d'une taille assez large.

12 Étant donné qu'il y a un décalage d'environ 10 ans entre la publication du corpus $\mathrm{Le}$ Monde et la publication des deux autres corpus, on pourrait se demander si les corpus sont tout à fait comparables. Selon nous, ceci ne pourrait influencer les résultats que dans une moindre mesure.

${ }^{13}$ Remarquons que le YCCQA est en fait un corpus multilingue, qui contient des données en français, en allemand, en anglais et en espagnol. Nous avons bien sûr uniquement eu recours au sous-corpus français.

${ }^{14}$ Des informations concernant le logiciel de concordance AntConc peuvent être retrouvées sur le site web suivant : http://www.laurenceanthony.net/software/antconc/.

${ }^{15}$ Selon certains linguistes, il n'est toutefois pas facile d'appliquer le modèle de Koch \& Oesterreicher $(1985,2007)$ à des données issues des « nouveaux médias » (p.ex. Dürscheid 2016).

${ }^{16}$ Le nombre de mots total des corpus est approximatif. En outre, pour le corpus Le Monde, il s'agit du nombre de mots lemmatisés. 
${ }^{17}$ Il y a 70 occurrences du marqueur au contraire et 198 occurrences du marqueur par contre dans le CFPP2000, mais trois occurrences ont été exclues. Il s'agit de trois exemples dans lesquels le marqueur au contraire ou par contre est répété plusieurs fois par le même locuteur :

(i) spk2 : ben moi j'ai gardé contact avec des gens d' collège et d' lycée + spk1 : de lycée que le passage en prépa euh ailleurs ça fait pas perdre spk2: non là pas du tout non au contraire + non au contraire $(\mathrm{mh})$ non non puis j'ai rencontré des nouveaux gens aussi donc euh (CFPP2000)

Dans ces cas, nous avons uniquement pris en considération la première occurrence (en caractères gras) ; la deuxième occurrence (soulignée) a été supprimée.

${ }^{18}$ Rappelons que notre corpus du français écrit formel contient uniquement les articles qui sont parus dans Le Monde en 1998 (voir 3). 